\title{
A CHEMOMECHANICAL NANOMACHINE FOR SENSING AND ACTUATION BASED ON A BIASED BROWNIAN RATCHET ARCHITECTURE
}

\author{
G. Lavella ${ }^{1}$, R. Morfino ${ }^{2}$, and M. M. Maharbiz ${ }^{1}$ \\ ${ }^{I}$ University of California, Berkeley, Berkeley, California, USA \\ ${ }^{2}$ Polytechnic University of Turin, Turin, Piedmont, Italy
}

\begin{abstract}
We present the design, fabrication and experimental demonstration of a self-powered transducer that couples molecular sensing and mechanical actuation into a single nanoscale structure. This capability is achieved through a chemomechanical device that uses an antibody based, biased Brownian ratchet architecture. We demonstrate that this new ratchet can directly convert a detection event into mechanical motion. The device can be tailored to specifically detect a broad range of molecules through the choice of antibody and operates in physiologic solutions. This work provides initial experimental evidence that this mechanism of chemomechanical transduction is feasible.
\end{abstract}

\section{INTRODUCTION}

Nanoscale machines which directly convert chemical energy into mechanical work are ubiquitous in nature and are employed to perform a diverse set of tasks such as transporting molecules, maintaining molecular gradients, and providing motion to organisms [1]. Numerous forms exist, categorized as biological motors, rotors, shuttles, springs and ratchets $[1,2]$. By and large, these devices are powered by energy extracted from concentration gradients, the breaking or formation of covalent bonds - most notably ATP, NADH, and NADPH - or in the case of ratchet type mechanisms from polymerization reactions [3]. In their own right, they operate as sensors and actuators, where the sensed molecule and the energy source are one and the same. The extensive study of their operation has provided scientists with empirical demonstrations of the mechanisms by which nanoscale machines can operate. They suggest that in a nanoscale environment where viscous and thermal forces dominate inertia, structures that perform chemomechanical transduction are well served by biasing random thermal movements with asymmetries in the energy landscape. This design strategy, which has been well understood by thermal physicists for decades [4], allows devices to accomplish directed motion against thermal fluctuations. Drawing from these lessons, we have developed a synthetic Brownian ratchet that converts antigenic binding into motion. However, unlike the Brownian machines observed in nature, our device extracts power from binding free energy by tensioning antibody-antigen, host-guest complexes. This shift in strategy enables chemomechanical transduction to be tailored to respond to a broad range of molecules and no longer limits it to a relatively small set of molecules, gradients or polymerization reactions. Throughout this paper we refer to our architecture as an antibody zipper.

It is instructive to begin by describing the basic structure of an antibody zipper. A schematic of the device used in our experiments can be seen in Figure 1. The structure is relatively simple; a precisely patterned backbone conjugated with a high density layer of active antibodies. It should be noted that the entire device is covered with antibodies; however, only antibodies in the region relevant to zippering are shown. Within a range of geometries, the operation of the device proceeds as follows. In solution, the structure experiences small vibrations in each arm from thermal fluctuations and ambient noise. When no antigen is present, no closing is observed (State 1 in Figure 1). As antigenic molecules are encountered, the antibodies on the top and bottom arms begin to bind antigen until a critical threshold is reached. We believe this threshold is determined by numerous kinetic, geometric and mechanical factors. Nevertheless, for this threshold to be reached it is necessary that a sufficient number of complexes (antibody-antigen) are created to enable sandwich bond (antibody-antigen-antibody) formation with vacant paratopes on an opposing arm (State 2 in Figure 1). The motion required to bring the two species into contact is supplied by thermal and ambient vibrations. In order to prevent saturation of antibodies with ligand before sandwich bonds can be formed, the kinetics associated with zippering must be faster than the antibody-antigen reaction kinetics. The resultant sandwich bonds that form across the arms prevent the device from relaxing back to its original unbound equilibrium state. This process repeats itself and the device zippers progressively from the hinge side towards the open end of the device, essentially zippering the device closed (State 3 in Figure 1).

State 1: Open (no antigen)

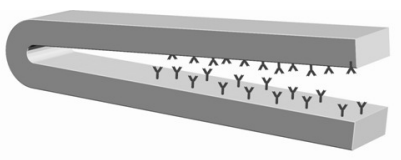

State 2: Zippering begins (antigen detection and loading)

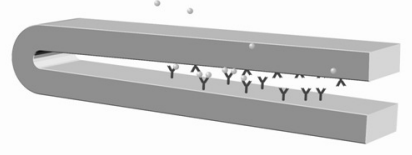

State 3: Closed (zippering complete)

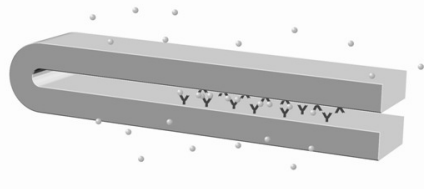
Detected Antigen: Antibody (lgG): $\mathbf{Y}$

Figure 1. Operational schematic for an antibody based Brownian Ratchet. Note the IgG layer covers the entire device.

\section{THEORY}

Brownian ratchet mechanisms are an area of active interest in the physics community. The detailed mechanisms by which they operate are diverse, however in all such devices, a random input (which need not be thermal), a source of energy and an asymmetry in the potential must be present. Tailoring these components such that the movement of a Brownian particle is statistically favored in one direction is critical to Brownian ratchet design. The potential energy surface of device reflects the design of system and can be used to understand which parameters must be altered in order to achieve a working device. The barrier heights dictate the amount of random input energy 
required for a Brownian particle to proceed, while the shape of the curve reflects both the asymmetry in the system and the energy input. The exact nature of this surface for our device as constructed is still under investigation.

Thermal and ambient vibrations act as the required randomization element and supply the necessary displacement of the Brownian particle. In our system this particle can be chosen arbitrarily as any point along the structural backbone. As thermal vibrations provide motion, sandwich bonds can form across the arms of the device. At this point, no energy has been transferred to the system. As the arms relax back to their equilibrium state, the sandwich bond is placed in tension and work is performed on the system. This work arises from the free energy change associated with the product of force and displacement along the reaction coordinate [5].

\section{RESULTS}

In this study, the devices used were bound to a substrate bound anchor with a small metal bridge. The devices are suspended $\sim 350 \mathrm{~nm}$ above the substrate. The interior angles at the hinge region ranged from $4^{\circ}$ to $6.9^{\circ}$. Both the arms and hinge were patterned to be approximately $50 \mathrm{~nm}$ in width and $30 \mathrm{~nm}$ thick. Device sets with arm lengths of $1 \mathrm{um}$ and 2 um were used.

Figure 2 shows micrographs of devices functionalized to detect streptavidin both before and after the introduction of the sensed molecule $(0.5 \mathrm{mg} / \mathrm{ml}$ streptavidin). The structural change is clearly noticeable in the active devices (Figure 2.a) and is not seen in the controls (Figure 2.b). Experiments for both active devices $(n=21)$ and controls $(n=23)$ were performed in parallel under identical conditions (immersed in phosphate buffered saline $\mathrm{pH} 7.4,25^{\circ} \mathrm{C}$ ).
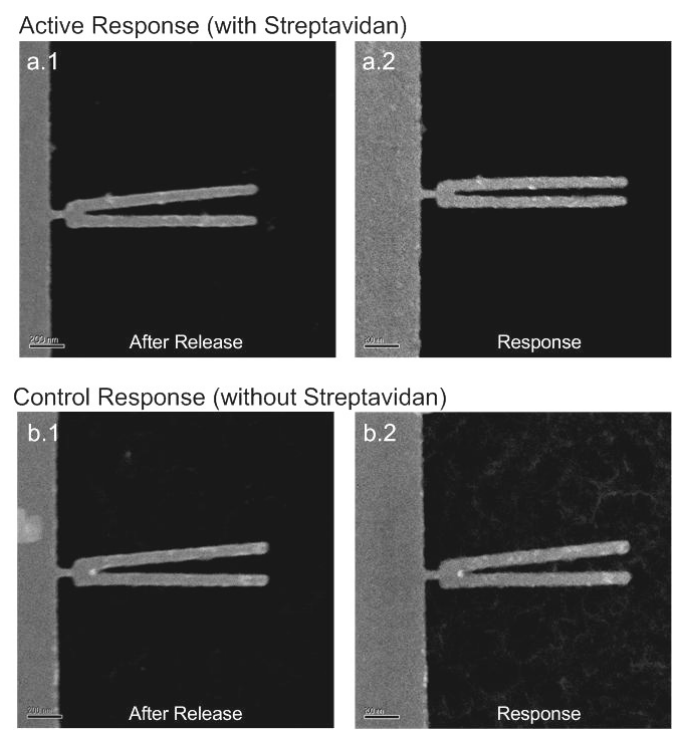

Figure 2. SEM images of anchored device response; a.1) freely suspended device before functionalization and introduction of streptavidin; a.2) response after functionalization and introduction of streptavidin into solution; b.1) control, freely suspended device before functionalization; b.2) control device, response after functionalization, no streptavidin was introduced into the solution.

Figure 3 shows a statistical distribution for the degree of closing in terms of 'percent closed' for the controls and active devices. Percent closed is computed by dividing the total deflection between tips of each arm by the deflection required to reach a closed state. We define a closed state as a distance between arms of $40 \mathrm{~nm}$ - the aggregated length of the sandwich bond and protein A stack.

\section{$\times$ Control Devices $(n=23) \quad \diamond$ Active Devices $(n=21)$}

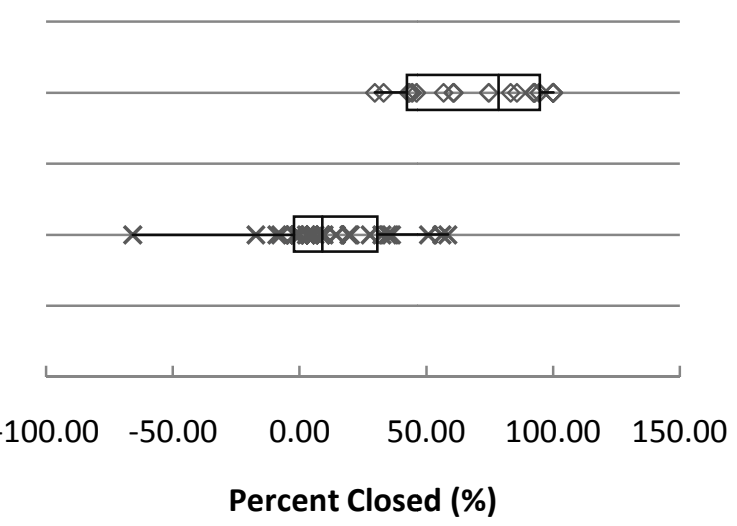

Figure 3. Antibody based, Brownian Ratchet response. Figure shows the statistical distribution of closing in active (median $=$ $83.5 \%$ ) and control (median $=9.72 \%$ ) devices.

\section{METHODS}

Devices were fabricated on silicon wafers. The wafers were first coated with poly-germanium layer via sputtering $(350 \mathrm{~nm}$, $\mathrm{R}_{\mathrm{rms}}<2 \mathrm{~nm}$ ). A resist bilayer (PMMA A2-950k/A3-50k, $40 \mathrm{~nm} / 70 \mathrm{~nm}$, Microchem) was then deposited by spin coating and patterned using electron beam lithography (Crestec CABL9510CC High Resolution Electron Beam Lithography System) and developed (MIBK/IPA 1:3, Microchem) for 60s at room temperature. Subsequently, a $\mathrm{Cr} / \mathrm{Au}(5 \mathrm{~nm} / 30 \mathrm{~nm})$ metal layer was directionally deposited by electron beam evaporation. Metal liftoff was then performed in acetone at room temperature. This resulted in devices anchored to the poly-germanium layer. The devices were then released by under-etching the polygermanium layer in $10 \% \mathrm{H}_{2} \mathrm{O}_{2}$ for ten minutes at room temperature. Devices used in these studies were prepared freely suspended over the substrates (as seen in Figure 2).

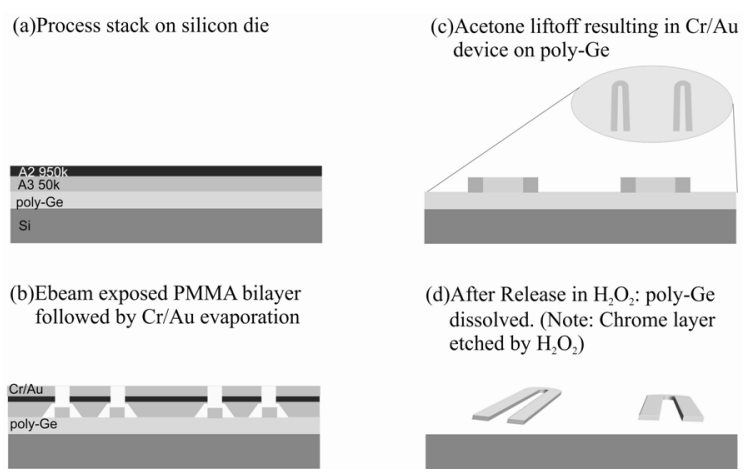

Figure 4. Process sequence for fabrication of gold structural backbone

After creating the gold structural backbone, the devices were washed in acetone $(3 \mathrm{x}, 5 \mathrm{~min})$ then in DI $(3 \mathrm{x}, 5 \mathrm{~min})$. The DI was then replaced by the organic solvent Dimethyl sulfoxide 
(DMSO) though a series of three washing steps. A $0.002 \mathrm{M}$ Dithio-bis(Succinimidyl Propionate) (DSP) in DMSO solution was prepared. DSP is a homobifunctional crosslinker molecule which binds to Protein A (Recombinant Staphylococcal) via the amine residues - at neutral $\mathrm{pH}$ or higher - and to the gold surface via the sulfide exposed during cleaving in DMSO. The solution containing the devices was then combined with a DSP solution and incubated for $2 \mathrm{hrs}$ at room temperature. Unbound DSP was removed from the solution by washing $(3 \mathrm{x}, 5 \mathrm{~min})$ in phosphate buffered saline pH 7.4 (PBS 7.4). A solution containing $0.1 \mathrm{mg} / \mathrm{ml}$ to $0.5 \mathrm{mg} / \mathrm{ml}$ of polyclonal $\mathrm{IgG}$ (rabbit anti-streptavidin, GenScript Corporation) was prepared, and added to the solution containing the devices and incubated for 1 $\mathrm{hr}$ at room temperature. Unbound $\operatorname{IgG}$ was removed through a series of three washing steps in PBS. BSA (1\% in PBS) was then added to the solution to block residual reactive sites and incubated for $1 \mathrm{hr}$. This method produced an oriented and densely packed polyclonal IgG layer. A high active $\mathrm{IgG}$ density was critical to proper device function. Two conjugation methods were explored, the first method directly cross-linked the $\operatorname{IgG}$ to the DSP monolayer, while the second method made use of Protein A (Pr A) intermediate layer to orient the antibodies, as describe in the method above. The results quantifying the active IgG density for both methods (Table 1) were obtained using Immuno-SEM and verified using quartz crystal microbalance (QCM).

Table 1. Comparison of active IgG functional densities measured on evaporated $\mathrm{Cr} / \mathrm{Au}$ substrates using amine crosslinked and Protein A conjugation

\begin{tabular}{|l|c|c|c|}
\hline Conjugation Method & $\begin{array}{c}\text { Functionalization } \\
\text { Molecules }\end{array}$ & $\begin{array}{c}\text { Density } \\
\text { [particles/cm2] }\end{array}$ & $\begin{array}{c}\text { Spacing between } \\
\text { active antibodies } \\
{[\mathrm{nm}]}\end{array}$ \\
\hline Amine Crosslinked & $\begin{array}{c}\mathrm{DSP}(0.002 \mathrm{M}), \mathrm{IgG} \\
(0.1 \mathrm{mg} / \mathrm{ml})\end{array}$ & 690 & 57.4 \\
\hline Protein A conjugated & $\begin{array}{c}\mathrm{DSP}(0.002 \mathrm{M}), \\
\operatorname{PrA}(0.5 \mathrm{mg} / \mathrm{ml}), \\
\mathrm{IgG}(0.1 \mathrm{mg} / \mathrm{ml})\end{array}$ & 1156 & 44.3 \\
\hline
\end{tabular}

After preparation, the device response to the antigenic biomolecule was measured. In parallel, a set of controls and active devices were prepared. All experiments were performed at room temperature. A PBS 7.4 solution containing $0.5 \mathrm{mg} / \mathrm{ml}$ streptavidin was introduced to solution containing the active devices, while in the controls only PBS 7.4 was introduced. The device sets were then incubated for 30 minutes. Following incubation, both device sets were washed in DI $(3 \mathrm{x})$. Both active and control device sets were then fixed in a $2 \%$ solution of glutaraldehyde for 30 minutes. The fixation agent was then removed in a series of washing steps, first in PBS 7.4 (3x, $5 \mathrm{~min})$, then in DI $(3 \mathrm{x}, 5 \mathrm{~min})$. The devices were then suspended in methanol and critically point dried (Tousimis 915B Critical Point Dryer). Micrographs of dry devices were then captured and analyzed.

\section{CONCLUSIONS}

Given the large set of molecules against which antibodies can be raised, and the innately high specificity of antibody binding, we believe this work could have broad applications in biological sensing and transduction. Specifically, we envision a class of nanodevices which can elute drugs and construct synthetic chemical signaling cascades through the coupling of a chemically-triggered mechanical event with the release of sequestered compounds in conjugated capsules. We believe mesoscale devices based on these architectures offer a paradigm shift for sensing and actuation inherent in the archicetecture which leverages mechanochemical phenomena across multiple size scales and that this will lead to the creation of new families of sensors, actuators and nanomachines.

\section{REFERENCES}

[1] L. Mahadevan and P. Matsudaira, "Motility Powered by Supramolecular Springs and Ratchets", Science, 288, 95 (2000) [2] E.R. Kay, D. A. Leigh, and F. Zerbetto, "Synthetic Molecular Motors and Mechanical Machines", Angew. Chem. Int. Ed., 46, 72-191 (2007)

[3] M.Muller, A. Engel, U. Aebi, "Structural and Physicochemical Analysis of the Contractile MM Phage Tail and Comparison with the Bacteriophage T4 Tail", J. Struct. Biol., 112, 1 (1994)

[4] R. Feynman, The Feynman Lectures on Physics

[5] C. Bustamante, Y. R. Chemla, N. R. Forde, and D. Izhaky, "Mechanical Processes in Biochemistry", Annu. Rev. Biochem, 73, 705-48 (2004) 